\title{
Displaying Economic Value
}

\author{
CAREN MARZBAN \\ Applied Physics Laboratory, and Department of Statistics, University of Washington, Seattle, Washington
}

(Manuscript received 8 November 2011, in final form 25 May 2012)

\section{ABSTRACT}

\begin{abstract}
The distinction between forecast quality and economic value in a cost-loss formulation is well known. Also well known is their complex relationship, even with some instances of a reversal between the two, where higher quality is associated with lower economic value, and vice versa. It is reasonable to expect such counterintuitive results when forecast quality and economic value-both, multifaceted quantities-are summarized by single scalar measures. Diagrams are often used to display forecast quality in order to better represent the multidimensional nature of forecast quality. Here, it is proposed that economic value be displayed as a region on a plot of hit rate versus false alarm rate. Such a display obviates any need to summarize economic value by a scalar measure. The choice of the axes is motivated by the relative operating characteristic (ROC) diagram, and, so, this manner of displaying economic value is useful for deterministic as well as probabilistic forecasts.
\end{abstract}

\section{Introduction}

The general situation involving a binary event, and binary or probabilistic forecasts of the event, is thoroughly studied (Jolliffe and Stephenson 2003; Murphy 1991, 1993; Wilks 2006). The quality of such forecasts is a multifaceted quantity (Murphy and Winkler 1987, 1992), and, therefore, information regarding forecast quality is lost when it is summarized by a single, scalar measure. For example, the probability of detection (or hit rate) and the false alarm rate individually provide an incomplete assessment of forecast quality. A high hit rate (suggesting high quality) may be accompanied by a high false alarm rate (suggesting low quality), or vice versa. Focusing on any single measure can lead to a completely false assessment of the true quality of forecasts, at worse, and, at best, it can lead to measures with undesirable properties under certain circumstances (Marzban 1998).

The multifaceted nature of forecast quality calls for a paradigm where diagrams take the place of a single measure of quality. For probabilistic forecasts, attribute diagrams, refinement diagrams, discrimination plots, and relative operating characteristic (ROC) curves are used

Corresponding author address: Caren Marzban, Dept. of Statistics, and Applied Physics Laboratory, University of Washington, Box 354322, Seattle, WA 98195-4322.

E-mail: marzban@stat.washington.edu to display different facets of forecast quality. For categorical (deterministic) forecasts, a recent proposal includes the performance diagram (Roebber 2009; Taylor 2001), where multiple scalar measures are plotted on a single diagram. Although there are differences between these diagrams, what is common to them is that they acknowledge the importance of displaying forecast quality in a multidimensional fashion (i.e., via a diagram).

Another well-studied problem involves the situation when a binary decision or action is to be based on forecasts (Doswell and Brooks 1998; Katz and Murphy 1997; Mason 2004; Richardson 2000; Wandishin and Brooks 2002; Wilks 2001, 2006). The concept that arises from considering such problems is the economic value of the forecasts. The economic value and the quality of forecasts are different facets of forecast goodness, often with a complex relationship between them (Murphy and Ehrendorfer 1987; Roebber and Bosart 1996).

Assessing economic value (henceforth value) requires a specification of the costs and losses incurred in taking, or not taking, an action based on the forecasts. However, not all decision problems lend themselves to a cost-loss analysis. For example, Stewart et al. (2004) discuss the value of precipitation forecasts with respect to snow removal. They show that the decision-making process in that problem is too complicated for a simple cost-loss analysis. And even when the problem is simplified, they find that the unavailability of appropriate data can

DOI: 10.1175/WAF-D-11-00138.1 
preclude a proper assessment of value. In the following, it is assumed that the decision process does allow for a cost-loss analysis.

The importance of examining the value of forecasts has been highlighted in a wide range of practical problems. Palmer (2002) compares three different precipitation forecasting systems and shows that ensemble systems have higher value than deterministic forecasts. Within the context of terminal aerodrome forecasts (TAFs), Keith (2003) has shown that airlines can benefit, through lower fuel usage, when the value of TAFs is taken into account. He shows that for some flights, even moderate quality forecasts can provide most of the economic savings gained by perfectly reliable TAFs. Keith and Leyton (2007) show that the value of probabilistic forecasts at airports can be used to determine the optimal amount of fuel to be carried by an airplane. The value of hurricane forecasts is taken into account by Letson et al. (2007) when they compare the benefits from multiple actions, for example, "improved forecast provision and dissemination vs. alternative public investments such as infrastructure or forecasts of other hazards." The value of wind forecasts has been examined for utility industries (Milligan et al. 1995), and Teisberg et al. (2005) have analyzed the value of temperature forecasts in electricity generation. All of these studies demonstrate the usefulness of examining the value of forecasts in conjunction with their quality.

In its simplest realization, value, like quality, is summarized by a single measure, which is plotted as a function of a quantity depending on the costs and losses. Also like quality, value is a multifaceted notion, and so information is lost when it is summarized by a single measure. Thus, neglecting the multidimensional nature of value can lead to false conclusions. At worse, it can lead one to believe that forecasts have high value, when in fact, they do not. Or, the forecasts may be declared to have little value, when in reality they have high value. Neglecting the multifaceted nature of value can also lead to counterintuitive or apparently contradicting conclusions. Indeed, a reversal of quality and value has been noted in the literature, where higher quality is associated with lower value, or vice versa (Murphy and Ehrendorfer 1987). This type of counterintuitive result can be explained by a number of arguments, including one proposed by Mason (2004) where the culprit behind the unexpected result is attributed to a nonoptimal probability threshold. For deterministic forecasts, an alternative explanation is that the aforementioned reversal occurs because multifaceted quantities (i.e., quality and value) are summarized by single, scalar measures. Avoiding a scalar measure of value not only precludes such counterintuitive conclusions but also provides a more complete, and therefore more useful, representation of the value of forecasts.

In this paper it is proposed that the value of binary (deterministic) forecasts be displayed as a region on a plot of the hit rate versus the false alarm rate, without the need for a scalar summary measure for value at all. Such a plot is of course the "background" upon which the ROC curve is drawn, and, so, the proposed method of displaying value is useful for probabilistic and deterministic forecasts alike.

\section{Forecast quality and economic value}

Denote the occurrence or nonoccurrence of an event with a 1 or 0 , respectively, and similarly, for taking an action (1), or not taking an action (0). Let $H$ and $F$ represent the hit rate and the false alarm rate, respectively. A commonly used (scalar) measure of forecast quality, specifically of discrimination, is the true skill score (TSS), also known by many other names (Wilks 2006):

$$
\mathrm{TSS}=H-F
$$

In the cost-loss formulation, an often-used toy model for the loss matrix is (Berger 1985; Katz and Murphy 1997)

$$
\begin{array}{r}
\text { Action } \\
\text { Event } \frac{0}{1}\left(\begin{array}{cc}
0 & C \\
L & L_{m}
\end{array}\right) .
\end{array}
$$

In this model, if an event does not occur, and no action is taken (action $=0$ ), then no cost or loss is incurred. If an event does not occur, but action is taken (action $=1$ ), then a cost $C$ is incurred. If an event does occur, and no action is taken, then the user loses an amount L. Finally, if an event occurs, and an action is taken, then a loss of $L_{m}$ is incurred. Note that the choice of the loss matrix is completely independent of forecasts or their quality.

The following quantities are central to ascertaining the value of forecasts (Katz and Murphy 1997; Wilks 2006):

$$
\begin{aligned}
E_{0} & =[\text { Expected cost if Action }=0]=p L, \\
E_{1} & =[\text { Expected cost if Action }=1]=(1-p) C+p L_{m}, \\
E_{f} & =[\text { Expected cost if Action }=\text { forecast }] \\
& =(1-p) F C+p(1-H) L+p H L_{m}, \\
E_{p} & =[\text { Expected cost if Action }=\text { perfect forecasts }]=p L_{m}, \\
E_{r} & =[\text { Expected cost if Action }=\text { random forecasts }] \\
& =(1-p) F_{1} C+p F_{0} L+p F_{1} L_{m},
\end{aligned}
$$


where $p$ is the prior (climatological) probability of the occurrence of an event, and $F_{0}$ and $F_{1}$ are the proportions of forecasts of " 0 " and " 1 ", respectively. In the absence of forecasts, it is self-evident that an action should be taken if it leads to a lower expected cost. Defining the ratio ${ }^{1}$

$$
\mathrm{Cl}=\frac{C}{C+L-L_{m}}
$$

then the first two equations in Eq. (3) imply

Take action if $p>\mathrm{Cl}$; otherwise, do not act.

In other words, from an economic point of view, and without any forecasts, it is beneficial to always take action if the probability of an event exceeds the ratio of cost and loss appearing in $\mathrm{Cl}^{2}$

A popular scalar measure of forecast value is (Richardson 2000; Wilks 2001)

$$
\begin{aligned}
V= & \frac{\text { Expected savings from forecasts }}{\text { Expected savings from perfect forecasts }}=\frac{\min \left(E_{0}, E_{1}\right)-E_{f}}{\min \left(E_{0}, E_{1}\right)-E_{p}}, \\
= & \frac{\min \left[p, \frac{C}{L}-p\left(\frac{C-L_{m}}{L}\right)\right]-p-(1-p)(F) \frac{C}{L}+p(H)\left(1-\frac{L_{m}}{L}\right)}{\min \left[p, \frac{C}{L}-p\left(\frac{C-L_{m}}{L}\right)\right]-p \frac{L_{m}}{L}} .
\end{aligned}
$$

Although this is normally the way $V$ is written, it simplifies considerably when written for $p<\mathrm{Cl}$ and $p>\mathrm{Cl}$ separately:

$$
V=\left\{\begin{array}{l|l}
H-R F & \text { if } p<\mathrm{Cl}(\text { i.e., } R>1) \\
(1-F)-\frac{1}{R}(1-H) & \text { if } p>\mathrm{Cl}(\text { i.e., } R<1)
\end{array}\right.
$$

where the quantity $R$ is defined as ${ }^{3}$

$$
R=\left(\frac{1-p}{p}\right)\left(\frac{\mathrm{Cl}}{1-\mathrm{Cl}}\right)
$$

Note that

$$
p<\mathrm{Cl} \Leftrightarrow R>1 \text { and } p>\mathrm{Cl} \Leftrightarrow R<1
$$

If $p=\mathrm{Cl}$ (i.e., $R=1$ ), then $V=H-F=\mathrm{TSS}$. In other words, if $p=\mathrm{Cl}$, then $V$ (i.e., a measure of value) reduces to a measure of quality.

\footnotetext{
${ }^{1}$ Richardson (2000) denotes this quantity $\alpha$.

${ }^{2}$ If $\mathrm{Cl}>1$, then no action should be taken, independently of $p$, because the expected cost associated with no action is always lower than that associated with action. But if $0<\mathrm{Cl}<1$, then the optimal decision depends on the value of $p$. For this reason, only $0<\mathrm{Cl}<1$ is examined here.

${ }^{3}$ If both $p$ and $\mathrm{Cl}$ are very small (i.e., $p \ll 1$ and $\mathrm{Cl} \ll 1$ ), then $R \sim$ $\mathrm{Cl} / p$. With $L_{m}=L, \mathrm{Cl}$ becomes $C / L$. Some reported $C / L$ ranges are as follows: for orchardists, 0.02-0.05 (Murphy 1977); loading of fuel for airplanes, 0.01-0.12 (Leigh 1995); and winter road gritting, 0.125 (Thornes and Stephenson 2001).
}

\section{Reversal of quality and value}

The above presentation allows for a simple demonstration of the aforementioned reversal phenomenon noted in the literature (Mason 2004; Murphy and Ehrendorfer 1987). Both TSS and $V$ depend on $H$ and $F$, and so are best displayed on a plot of $H$ versus $F$. This choice of the variables across the $x$ and $y$ axes is the same as that of the ROC diagram (Fawcett 2006; Marzban 2004). On a plot of $H$ versus $F$, both TSS $=$ constant and $V=$ constant are straight lines. Figure 1 shows the (solid) lines TSS $=0.3$, TSS $=0.4$, and the (dashed) lines $V=0.1, V=0.2$. The former have slope 1 , while the slope of the latter is $R=1.6 .{ }^{4}$ Consider two forecasting systems corresponding to the filled and open circles in Fig. 1. One has higher quality (TSS) than the other but lower value $(V)$. This type of reversal may seem concocted, but it is actually quite natural and is dictated by the geometry of two parallel lines intersecting another pair of parallel lines. Note that if $p=\mathrm{Cl}$ (i.e., $R=1$ ), then all lines have slope $=1$, and so no intersection between the lines can occur. In other words, this type of reversal occurs when $p \neq \mathrm{Cl}$.

\section{Value region}

Such a reversal of quality and value arises because they are summarized by single, scalar measures. The

\footnotetext{
${ }^{4}$ For small $p$ and $\mathrm{Cl}$, an $R$ of 1.6 corresponds to $p / \mathrm{Cl}=1 / R=$ 0.625 .
} 


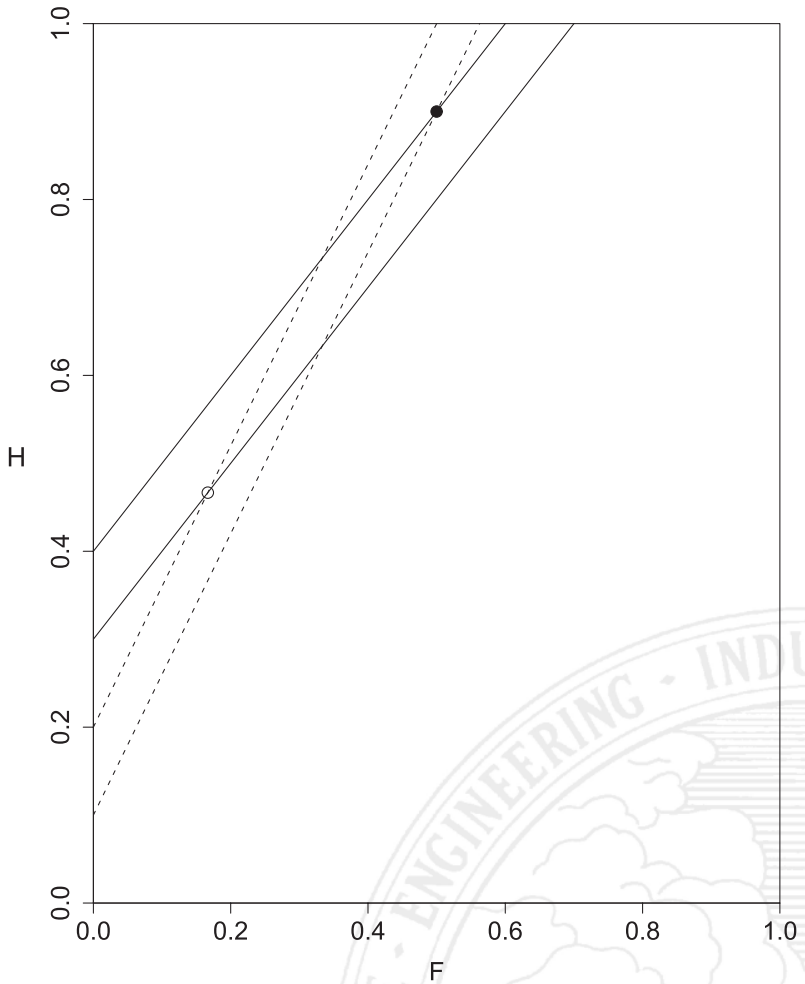

FIG. 1. A demonstration of the reversal of quality and value. The solid parallel lines correspond to TSS $=0.3$ and 0.4 , and the two dashed lines represent forecasts with $V=0.1$ and 0.2 . The forecasts corresponding to the filled circle have higher quality, but lower value than the forecasts associated with the open circle.

reason is that by virtue of being a scalar quantity, $V$ does not fully capture the multiple facets of value. But one can convey a more complete representation of value by returning to the expected costs in Eq. (3), from which $V$ is constructed. For example, it is reasonable to define valuable forecasts as those satisfying

$$
E_{p}<E_{f}<E_{0}, \quad E_{1}, \quad \text { and } E_{r} .
$$

In other words, the expected cost from using forecasts ought to be greater than that from perfect forecasts, but lower than either $E_{0}, E_{1}$, and the cost associated with acting according to random forecasts. Here, Eq. (10) defines economically valuable forecasts. ${ }^{5}$

In light of the expected costs in Eq. (3), the inequalities in Eq. (10) become

$$
L_{m}<L, \quad \frac{H}{F}>R, \quad \frac{1-H}{1-F}<R, \quad H>F .
$$

\footnotetext{
${ }^{5}$ The less than sign in Eq. (10) may be changed to less than or equal to but not much is gained from that revision.
}

The left-most constraint is trivial in that it is satisfied on physical grounds. It is easy to show that the right-most constraint is implied by the middle two constraints, which together place a severe restriction on possible values of $H, F$, and $R$. These two constraints can be written as

$$
H>R F, \quad H>(1-R)+R F,
$$

and their linearity in $H$ and $F$ again allows for a simple representation on a diagram of $H$ versus $F$. For a given $p / \mathrm{Cl}$, only one of these constraints is nontrivial, depending on $p<\mathrm{Cl}$ (i.e., $R>1$ ) or $p>\mathrm{Cl}$, (i.e., $R<1$ ), respectively. Therefore, the region corresponding to valuable forecasts-termed "value region"-is a triangular region bounded below by the lines $H=R F$ or $H=(1-R)+R F$. To make a connection with the scalar measure of value, these lines correspond to $V=0$, depending on whether $p<\mathrm{Cl}$ or $p>\mathrm{Cl}$. The connection between the value region and $V=$ constant lines is entirely expected. The important point, however, is that value can be displayed without a scalar measure.

For a given $H$ and $F$, it is possible to solve Eq. (12) for the critical value of $R$ separating forecasts with value from those without value. The $R$ values corresponding to valuable forecasts are

$$
\frac{1-H}{1-F}<R<\frac{H}{F}
$$

These constraints on $R$ can be translated into constraints on $\mathrm{Cl}$, by virtue of Eq. (8). Forecasts with value must have

$$
\frac{1}{1+\left(\frac{1-p}{p}\right)\left(\frac{1-F}{1-H}\right)}<\mathrm{Cl}<\frac{1}{1+\left(\frac{1-p}{p}\right)\left(\frac{F}{H}\right)}
$$

Again, in order to make a connection with the scalar measure of value, $V$, the interval specified in Eq. (14) is where $V$ is nonnegative. It is worth pointing out that Richardson (2000) shows that the quantities appearing in Eq. (14) are the conditional probability of the occurrence of an event, given a forecast of "yes" or "no," respectively. The particular form in Eq. (14) (not written in terms of these conditional probabilities) is intended to distinguish $F$ and $H$, which assess forecast quality, from $p$, which is determined by climatology.

The value region for different values of $p$ and $\mathrm{Cl}$ is shaded in gray in Fig. 2. The specific values of $p$ and $\mathrm{Cl}$ selected here are $0.001,0.008,0.018,0.279$, and 0.99 (motivated by the "Finley data," described in the next paragraph.) Only forecasts whose hit rate and false alarm rate fall in the shaded region are economically valuable. Also, note that the extent of the value region is 
JOBNAME: WAF 00\#0 2012 PAGE: 5 SESS: 8 OUTPUT: Fri Aug 24 15:34:56 2012 Total No. of Pages: 9

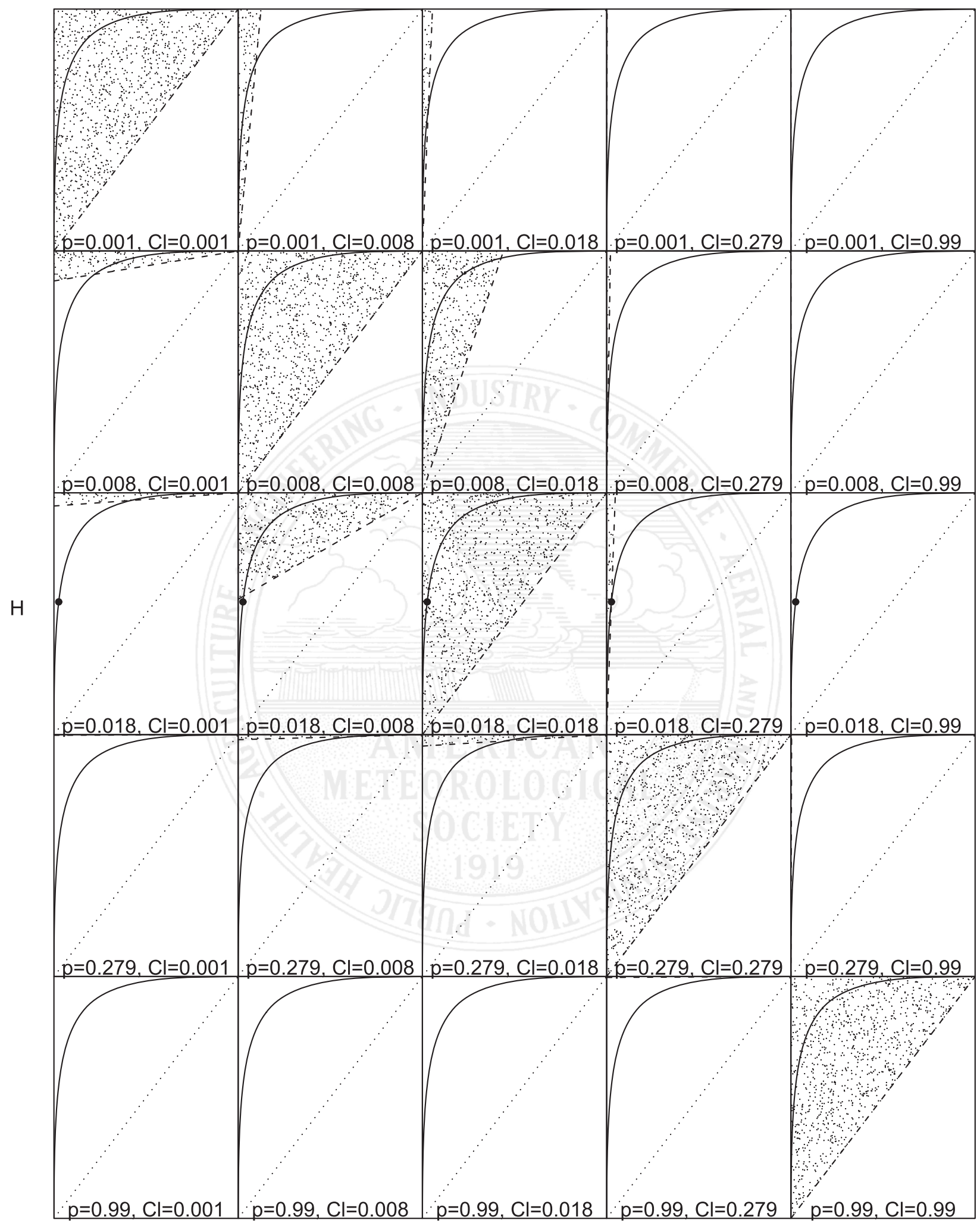

$\mathrm{F}$

FIG. 2. The value region (shaded) for different values of $p$ and $\mathrm{Cl}$. The filled circle corresponds to the Finley dataset. The arched curve is an example of an ROC curve based on a binormal model whose parameters have been chosen so that the ROC curve goes through the Finley point. 
TABLE 1. The Finley dataset.

\begin{tabular}{lcc}
\hline \hline & No tornado forecast & Tornado forecast \\
\hline No tornado observed & 2680 & 72 \\
Tornado observed & 23 & 28 \\
\hline
\end{tabular}

maximum when $p$ and $\mathrm{Cl}$ are comparable. For situations when they differ significantly, the value region is relatively small. This is a reflection of the fact that taking action, and not taking action, are the optimal decisions when $p \gg \mathrm{Cl}$ and $p \ll \mathrm{Cl}$, respectively.

As a concrete example, consider the Finley data (Murphy 1996) whose contingency table is shown in

T1 Table 1. One has $H=0.549, F=0.026$, TSS $=0.523$, and $p=0.018$. The filled circle in the $p=0.018$ panels in Fig. 2 corresponds to this data. The manner in which it falls inside, on the boundary, or outside the value region, depending on $\mathrm{Cl}$, is evident. Indeed, according to Eqs. (13) and (14), Finley forecasts have value only if $0.463<$ $R<21.11$ or $0.0084<\mathrm{Cl}<0.279$. For users whose $\mathrm{Cl}$ falls outside of this range, the forecasts have no value at all, regardless of their quality. As such, the user should ignore the forecasts and simply either act, or not act, according to the prescription in Eq. (5). Again, note that no scalar measure of value has been summoned in this representation of value.

This display of value is useful even when forecasts are probabilistic, or of a type for which ROC curves can be generated. The panels in Fig. 2 also show an ROC curve based on a binormal model (Marzban 2004) going through the "Finley point.", It does not correspond to any "real" probabilistic forecasts of tornados. Its purpose is only to demonstrate the interplay between the ROC curve and the value region. For example, if $p \neq \mathrm{Cl}$ (i.e., away from the diagonal panels in Fig. 2), then the ROC curve has segments that do not fall in the value region. In other words, even though the underlying probabilistic forecasts clearly have high quality, reflected in the high arc of the ROC curve, or the large area under it, for some probability thresholds the resulting binary forecasts have no value. This is consistent with the arguments of Mason (2004) for being careful in selecting optimal thresholds when value is summarized by $V$.

\section{Uncertainty}

The value region is bounded below by a straight line whose slope and intercept are determined by $R$ only; see

\footnotetext{
${ }^{6}$ The means of the two normal distributions are -1.035 and +1.035 , and both standard deviations are 1 .
}

Eq. (12). As such, the only source of uncertainty with respect to the extent of the value region is $R$ itself. Given that $R$ is determined by $p$ and $\mathrm{Cl}$, the uncertainty in $R$, denoted $\delta R$, can be computed from uncertainty in the latter, denoted $\delta p$, and $\delta \mathrm{Cl}$, respectively. It is reasonable to assume that uncertainty in $p$ is independent of uncertainty in $\mathrm{Cl}$, because the former is estimated from data, while the latter depends on the specifics of a user. If this assumption is valid, then Eq. (8) implies

$$
\left(\frac{\delta R}{R}\right)^{2}=\frac{1}{(1-p)^{2}}\left(\frac{\delta p}{p}\right)^{2}+\frac{1}{(1-\mathrm{Cl})^{2}}\left(\frac{\delta \mathrm{Cl}}{\mathrm{Cl}}\right)^{2}
$$

This equation allows one to compute the uncertainty in $\mathrm{R}$ from uncertainties in $p$ and $\mathrm{Cl}$. To simplify further, one may assume that events and nonevents occur independently (a poor assumption), in which case $(\delta p)^{2}$ can be measured by the variance of $p$, that is, $p(1-p) / N$, where $N$ is the total number of events and nonevents in the sample. Under this assumption, the first term in Eq. (15) is inversely proportion to $N$, and so it will be negligible relative to the second term, for a sufficiently large sample size. In that case, Eq. (15) simplifies to $\delta R / R=[1 /(1-\mathrm{Cl})](\delta \mathrm{Cl} / \mathrm{Cl})$. So, for a sufficiently large sample, this simple formula can readily compute the uncertainty in $R$ from the uncertainty in $\mathrm{Cl}$ itself.

The visual effect of uncertainty is to broaden the lower boundary line of the value region into a triangular region. This is shown in Fig. 3, when $p<\mathrm{Cl}$ (left panel) and $p>\mathrm{Cl}$ (right panel). In these figures, and for purely visual purposes, the specific uncertainty in $p$ is \pm 0.5 . Also shown in these figures, only as a point of reference, is the Finley point along with its uncertainty in both the $x$ and $y$ directions. Given that HR and FR are proportions, and assuming independence of daily tornadic activity (again, a poor assumption), their uncertainty is proportional to the standard deviation of a proportion, based on a sample of size $n$ (i.e., $\sqrt{p(1-p) / n}$ ). The "cross" atop the Finley point in Fig. 3 is \pm 2 times this standard deviation. The horizontal segment of the cross is much smaller than the vertical segment, because, as evidenced in the Finley dataset (Table 1), the sample size from which FR is computed (i.e., $2680+72$ ) is much larger than that upon which HR is based $(23+28)$. Still, these figures illustrate how uncertainty plays into the value region: the HR and FR (as well as their uncertainty) of forecasts will generally be represented by a cross, while the uncertainty in $p$ and $\mathrm{Cl}$ will lead to a broadening of the lower boundary of the value region. If a significant portion of the cross falls within the boundary region's "significant portion," then one may conclude that the value attributed to the forecasts is unlikely to be due to 

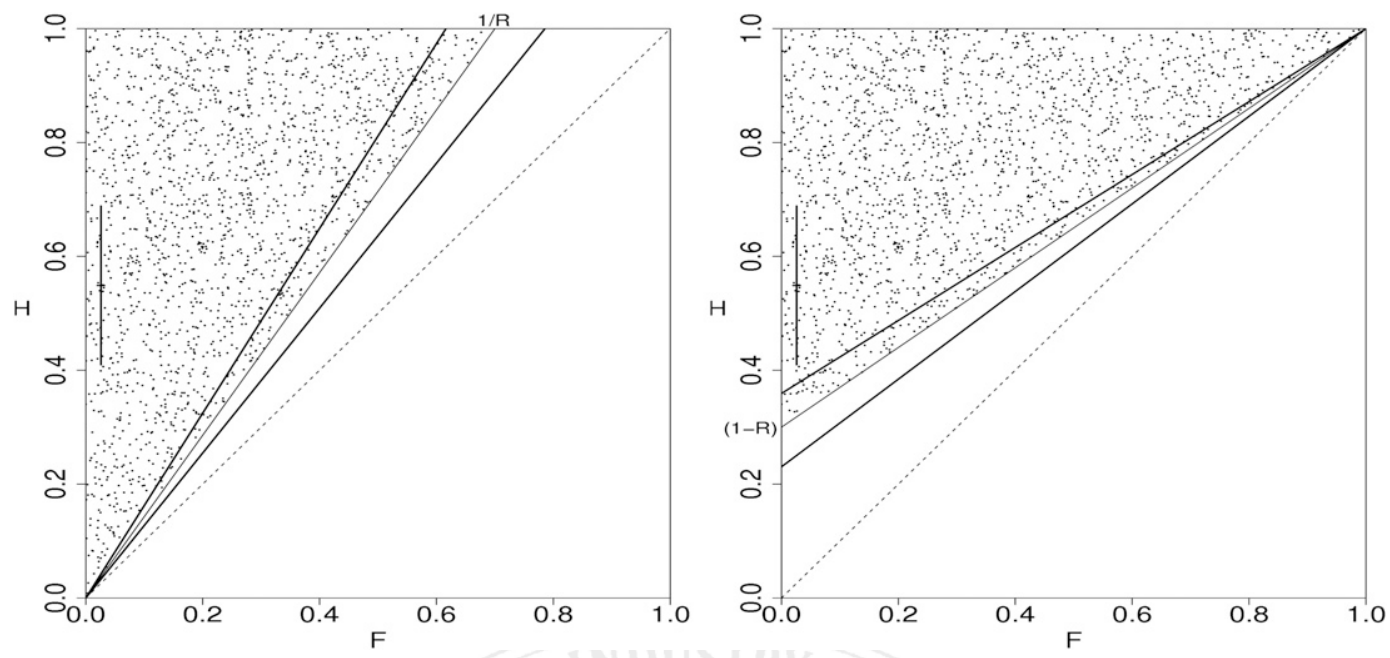

FIG. 3. The effect of uncertainty on the value region, when (left) $p<\mathrm{Cl}$ and (right) $p>\mathrm{Cl}$. The "cross" on the left side of the graph shows the Finley point and its uncertainty.

chance. Given that these displays are intended to provide information in a visual manner, and more information than that provided by a single number, it is unnecessary to quantify that (un)likelihood by a statistical test. In the cases displayed in Fig. 3, for example, one would be justified in concluding that the value is not due to chance. On the other hand, if a significant portion of the cross falls outside of the value region, then one can only conclude that the data do not provide sufficient information in support of any claim regarding the population/true value of economic value.

\section{Summary and discussion}

It is proposed that the (economic) value associated with a set of forecasts not be measured by a scalar quantity. It is argued that forecasts can be said to have value if the expected cost associated with actions linked to forecast satisfies some very general and reasonable inequalities. For example, actions linked to valuable forecasts ought to have a lower expected cost than actions based on random forecasts, or actions not based on forecasts at all. The inequalities define a value region, which is most naturally displayed in a diagram of the hit rate versus the false alarm rate (i.e., the "background" upon which the ROC curve is drawn). As such, quality and value can be displayed in a single diagram, without a summary measure for either. If a point on the ROC diagram falls within the value region, then the deterministic forecasting system can be said to have value. A consequence of using the value region is that one can no longer rank different forecasting systems, because ranking requires a scalar measure; all systems within the value region must be treated as equal in terms of their value. In this sense, the value region treats the value of forecasts as a binary quantity; forecasts either have value, or they do not. For probabilistic forecasts, or other forecasts for which an ROC curve can be produced, the portion of the ROC curve that falls within the value region is said to have value. In this way, some probability thresholds lead to valuable forecasts, and some do not. In addition to offering a more complete picture of value, use of the value region (as opposed to a scalar measure) also precludes counterintuitive conclusions such as the reversal of the relationship between quality and value. The value region is defined essentially by the equation of a straight line and is, therefore, extremely easy to compute without any sophisticated computer code. The formulas for computing the uncertainty in the value region are also simple to implement.

The connection between the value region and the scalar measure $V$ is simple: The former corresponds to all points on the ROC diagram for which the latter is nonnegative. This connection is not surprising because both concepts are based on the same set of expected costs. However, the value region carries more information by virtue of being a two-dimensional quantity. And displaying it on an ROC diagram, in particular, makes it especially useful given the ubiquity of ROC diagrams.

In addition to the works mentioned in the introduction, the connection between the ROC curve and expected cost has also been examined in fields outside of meteorology; two of these works are worth discussing here because of their close connection to the notion of the value region. Provost and Fawcett (1997) consider the slope of an "iso-performance line," which is the locus 
of points on an ROC diagram with equal expected cost $E_{f}$. Although they examine the situation where $L_{m}=0$, it is easy to show that the slope of their iso-performance line is exactly the slope of the constant value line, with value defined by $V$ in Eq. (7).

Drummond and Holte (2006) begin with the connection between cost (or loss) and the slope of a line on the ROC diagram but argue that the comparison of different forecasting systems is hampered by the visual effort to compare slopes of lines (e.g., tangent to the ROC curve). Instead, they consider an alternative diagram involving what they call "cost curves," defined as straight lines in "cost space," that is, a plot of expected cost versus $p L /[p L+(1-p) C] .^{7}$ ROC space and cost space are described as having a "dual" relationship in that the intercept and slope of a cost curve are determined by the coordinates of a point on the ROC diagram; similarly, a line on the ROC diagram translates to a point in cost space. The main advantage of cost curves over ROC curves is that the former make it easier to compare two different forecasting systems in terms of the expected cost associated with the forecasts. Another advantage is that confidence intervals for cost curves can be more easily displayed. The notion of a cost curve has many similarities to the notion of a value region proposed here, but there are some important differences. For example, the value region is displayed on an ROC diagram. In spite of all the advantages of cost curves over ROC curves, the latter are still useful and commonly employed. As such, displaying the value region adds useful information to the ROC diagram. Also, whereas cost curves are conducive to the comparison of two forecasting systems, the value region is useful even when examining a single forecasting system, that is, a single point on the ROC diagram (e.g., for deterministic forecasts) or a single ROC curve (e.g., for probabilistic forecasts).

Acknowledgments. We are grateful to Harold Brooks and Daniel S. Wilks for valuable comments. David Jones is acknowledged for providing the initial impetus for this work.

\section{REFERENCES}

Berger, J. O., 1985: Statistical Decision Theory and Bayesian Analysis. Springer-Verlag, 617 pp.

Doswell, C. A., III, and H. Brooks, 1998: Budget cutting and the value of weather services. Wea. Forecasting, 13, 206-212.

\footnotetext{
${ }^{7}$ The analysis in Drummond and Holte (2006) is based on a cost model where $L_{m}=0$, in which case the $x$ axis of the cost curve is equal to $1 /(1+R)$.
}

Drummond, C., and R. C. Holte, 2006: Cost curves: An improved method for visualizing classifier performance. Mach. Learn., 65, 95-130.

Fawcett, T., 2006: An introduction to ROC analysis. Pattern Recognit. Lett., 27, 861-874.

Jolliffe, I. T., and D. B. Stephenson, 2003: Forecast Verification: A Practitioner's Guide in Atmospheric Science. John Wiley and Sons, $240 \mathrm{pp}$.

Katz, R. W., and A. H. Murphy, 1997: Economic Value of Weather and Climate Forecasts. Cambridge University Press, $225 \mathrm{pp}$.

Keith, R., 2003: Optimization of value of aerodrome forecasts. Wea. Forecasting, 18, 808-824.

— of statistical probability forecasts for airports. Wea. Forecasting, 22, 928-935.

Leigh, R. J., 1995: Economic benefits of terminal aerodrome forecasts for Sydney airport, Australia. Meteor. Appl., 2, 239-247.

Letson, D., D. S. Sutter, and J. K. Lazo, 2007: Economic value of hurricane forecasts: An overview and research needs. Nat. Hazards Rev., 8, 78-86.

Marzban, C., 1998: Scalar measures of performance in rare-event situations. Wea. Forecasting, 13, 753-763.

- 2004: The ROC curve and the area under it as a performance measure. Wea. Forecasting, 19, 1106-1114.

Mason, I., 2004: The cost of uncertainty in weather prediction: Modelling quality-value relationships for yes/no forecasts. Aust. Meteor. Mag., 53, 111-122.

Milligan, M. R., A. H. Miller, and F. Chapman, 1995: Estimating the economic value of wind forecasting to utilities. Proc. Windpower '95, Washington, DC, National Renewable Energy Laboratory.

Murphy, A. H., 1977: The value of climatological, categorical and probabilistic forecasts in the cost-loss ratio situation. Mon. Wea. Rev., 105, 803-816.

- 1991: Forecast verification: Its complexity and dimensionality. Mon. Wea. Rev., 119, 1590-1601.

, 1993: What is a good forecast? An essay on the nature of goodness in weather forecasting. Wea. Forecasting, 8, 281293.

1996: The Finley affair: A signal event in the history of forecast verification. Wea. Forecasting, 11, 3-20.

— accuracy and value of forecasts in the cost-loss ratio situation. Wea. Forecasting, 2, 243-251.

— verification. Mon. Wea. Rev., 115, 1330-1338.

— , and — 1992: Diagnostic verification of probability forecasts. Int. J. Forecasting, 7, 435-455.

Palmer, T. N., 2002: The economic value of ensemble forecasts as a tool for risk assessment: From days to decades. Quart. J. Roy. Meteor. Soc., 128, 747-774.

Provost, F., and T. Fawcett, 1997: Analysis and visualization of classifier performance: Comparison under imprecise class and cost distributions. Proc. Third Int. Conf. on Knowledge Discovery and Data Mining, Newport Beach, CA, Association for the Advancement of Artificial Intelligence.

Richardson, D. S., 2000: Skill and relative economic value of the ECMWF Ensemble Prediction System. Quart. J. Roy. Meteor. Soc., 126, 649-667.

Roebber, P. J., 2009: Visualizing multiple measures of forecast quality. Wea. Forecasting, 24, 601-608. 
—, and L. F. Bosart, 1996: The complex relationship between forecast skill and forecast value: A real-world analysis. Wea Forecasting, 11, 544-559.

Stewart, T. R., R. Pielke Jr., and R. Nath, 2004: Understanding user decision making and value of improved precipitation forecasts: Lessons from a case study. Bull. Amer. Meteor. Soc., 85, 223-235.

Taylor, K. E., 2001: Summarizing multiple aspects of model performance in a single diagram. J. Geophys. Res., 106 (D7), 7183-7192.

Teisberg, T. J., R. F. Weiher, and A. Khotanzad, 2005: The economic value of temperature forecasts in electricity generation. Bull. Amer. Meteor. Soc., 86, 1765-1771.
Thornes, J. E., and D. B. Stephenson, 2001: How to judge the quality and value of weather forecast products. Meteor. Appl., 8, 307-314.

Wandishin, M. S., and H. E. Brooks, 2002: On the relationship between Clayton's skill score and expected value for forecasts of binary events. Meteor. Appl., 9, 455-459, doi:10.1017/ S1350482702004085.

Wilks, D. S., 2001: A skill score based on economic value for probability forecasts. Meteor. Appl., 8, 209-219.

_ 2006: Statistical Methods in the Atmospheric Sciences. Academic Press, 627 pp.

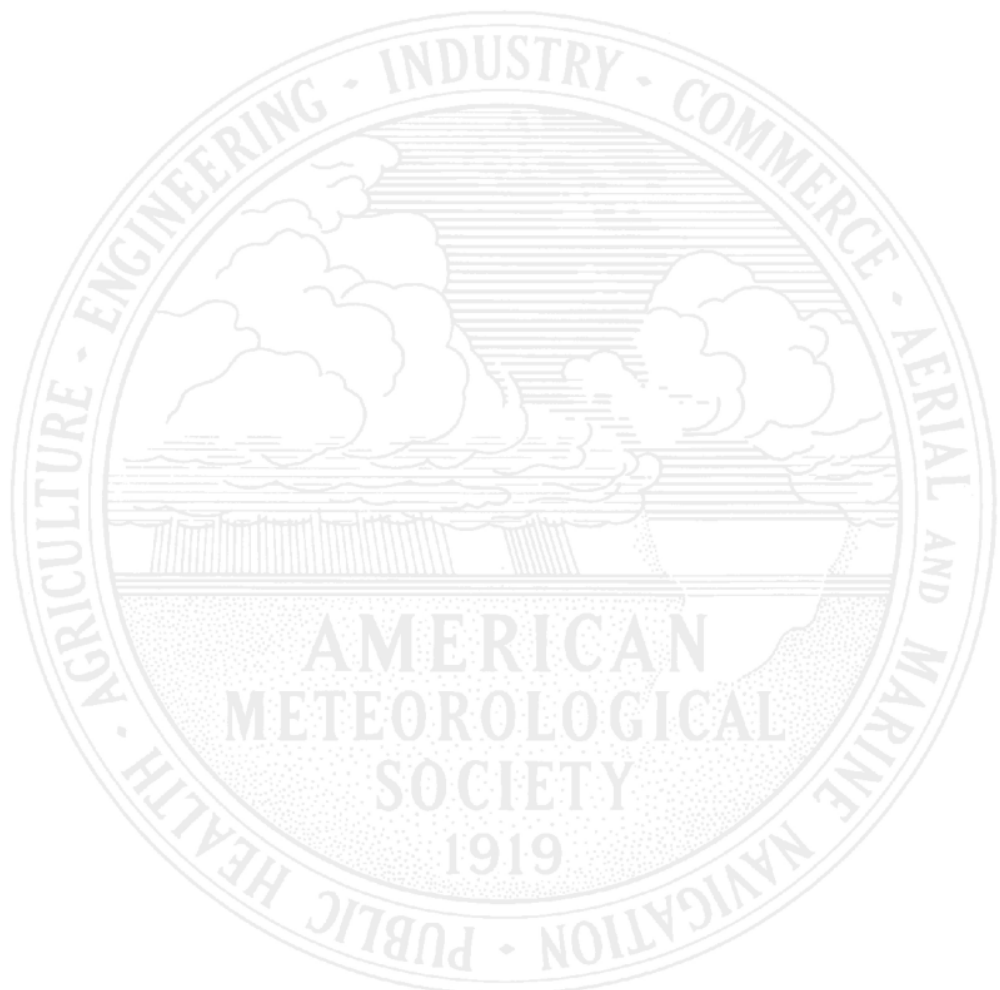

